\title{
Aprender conectados: un estudio sobre las redes personales de aprendizaje de estudiantes universitarios
}

\section{Connected learning: a study on university students' personal learning networks}

\author{
Jose Antonio García-Martínez ${ }^{1}$ \\ jose.garcia.martinez@una.cr \\ Graciela Herrera-Villalobos \\ graciela.herrera.villalobos@una.cr \\ Manuel Arturo Fallas-Vargas \\ manuel.fallas.vargas@una.cr \\ Universidad Nacional, Heredia, Costa Rica
}

\section{Resumen:}

Entender cómo aprenden los estudiantes en la actualidad es de vital importancia para la toma de decisiones. En este sentido, la incorporación de las TIC está modificando las dinámicas de enseñanza y aprendizaje tanto formales como no formales e informales. El objetivo de este estudio es analizar las interacciones a través de diferentes recursos tecnológicos que contribuyen a la formación de estudiantes universitarios. Los entornos personales de aprendizaje (PLE) son considerados como un entramado de herramientas, actividades, finalidades y conexiones que las personas utilizan para su aprendizaje. En este trabajo se retoman las redes personales de aprendizaje (PLN) como parte imprescindible de estos, destacando así el carácter social del aprendizaje. El estudio parte de un enfoque cuantitativo a través de un diseño no experimental y transaccional. La muestra $(n=1187)$ fue seleccionada de for-

\begin{abstract}
:
Understanding the ways in which students learn nowadays is of vital importance in order to make pedagogical decisions. In that sense, the incorporation of ICT is modifying formal, non-formal and informal teaching and learning dynamics. This study aims to analyse the interactions through different technological resources that contribute to the education of university students. Personal learning environments (PLE) are viewed as a set of tools, activities, aims and connections that people use in their learning processes. In this stu$\mathrm{dy}$, personal learning networks (PLN) are held as an indispensable part of them, thus highlighting the social nature of learning processes. The study takes a quantitative approach through a non-experimental and transactional design. The sample $(n=1187)$ was selected in a probabilistic way and targeted students from all programs at the Universidad Nacional (Costa Rica). Data
\end{abstract}

1 Dirección para correspondencia (correspondence address):

Jose Antonio García-Martínez. Campus Omar Dengo, Universidad Nacional. División de Educación para el Trabajo (CIDE). CP. 863000. Heredia, Costa Rica. 
Aprender conectados: un estudio sobre las redes personales de aprendizaje de estudiantes universitarios

Jose Antonio García-Martínez, Graciela Herrera-Villalobos y Manuel Arturo FallasVARGAS

ma probabilística y queda representada por estudiantes universitarios de los últimos años de todas las carreras de las Universidad Nacional (Costa Rica). La recogida de datos se llevó a cabo a través de un cuestionario elaborado ad hoc. Los resultados muestran que los estudiantes interactúan en mayor medida con los compañeros y el profesorado a través de la comunicación móvil y el correo electrónico. Destaca la escasa conexión con profesionales, así como el uso limitado de herramientas que permiten ampliar las redes de aprendizaje. Por otro lado, se han encontrado diferencias en las interacciones de acuerdo con el rendimiento académico, dejando entrever la importancia de enriquecer los PLE en general y las PLN en particular.

\section{Palabras clave:}

Red personal de aprendizaje; entorno personal de aprendizaje; interacciones; tecnologías de la información y la comunicación; estudiantes universitarios; educación superior. collection was carried out through a questionnaire developed ad hoc. The results demonstrate that students prefer to interact with their classmates and teachers via mobile communication and email. Connections with professionals is scarce as it is the use of tools through which learning networks are amplified. On the other hand, differences were reported in the interactions according to academic performance, pointing to the importance of enriching PLE in general and PLN in particular.

\section{Key words:}

Personal learning network; personal learning environment, interactions; information technology and communication; university students; higher education.

\section{Résumé:}

Comprendre comment les étudiants apprennent aujourd'hui est d'une importance capitale pour la prise de décision. En ce sens, I'incorporation des TIC modifie la dynamique de l'enseignement et de l'apprentissage formels, non formels et informels. L'objectif de cette étude est d'analyser les interactions à travers différentes ressources technologiques qui contribuent à la formation des étudiants universitaires. Les environnements d'apprentissage personnels (EAP) sont considérés comme un réseau d'outils, d'activités, de buts et de connexions que les gens utilisent pour leur apprentissage. Dans ce travail, les réseaux d'apprentissage personnels (PLN) sont repris comme une partie essentielle de ceux-ci, soulignant ainsi la nature sociale de l'apprentissage. L'étude est basée sur une approche quantitative à travers un design non expérimental et transactionnel. L'échantillon $(\mathrm{n}=1187)$ a été sélectionné de manière probabiliste et est représenté par des étudiants universitaires en dernière année de tous les cours de licence à l'Université nationale (Costa Rica). La collecte des données a été effectuée au moyen d'un questionnaire ad hoc. Les résultats montrent que les étudiants interagissent davantage avec leurs pairs et les professeurs par le biais de la communication mobile et du courrier électronique. La rareté des liens avec les professionnels ressort, de même que l'utilisation limitée des outils permettant d'étendre les réseaux d'apprentissage. D'autre part, on a constaté des différences dans les interactions en fonction des résultats scolaires, ce qui laisse entrevoir l'importance d'enrichir les PLE en général et les PLN en particulier.

\section{Mots clés:}

Réseau d'apprentissage personnel; environnement d'apprentissage personnel; interactions; technologies de l'information et de la communication; étudiants universitaires; enseignement supérieur.

Fecha de recepción: 16-01-2021

Fecha de aceptación: 20-03-2021 


\section{Introducción}

Durante las últimas décadas, las Tecnologías de la Información y de la Comunicación (TIC) han cobrado gran relevancia en la vida cotidiana de las personas, generando cambios profundos en el campo educativo (Ordóñez, Vázquez-Cano, Arias-Sánchez y López-Meneses, 2021) tanto en el desarrollo de procesos formales como informales y, favoreciendo paralelamente, una fructífera articulación entre el plano educativo y otros campos fundamentales que facilitan el bienestar integral de las personas. Es importante sugerir que dicha importancia, se debe en gran parte a la facilidad de acceso y difusión de la información (González Sanmamed, Muñoz-Carril y Santos-Caamaño, 2019), así como al gran abanico de posibilidades existentes que pueden facilitar los diferentes estilos y ritmos de aprendizaje (Uriguen-Aguirre, Vega-Jaramillo y Luna-Romero, 2020). Estos aspectos han quedado especialmente evidenciados ante la situación de pandemia ocasionada por el COVID-19, la cual ha resignificado drásticamente la metodología en los procesos de enseñanza-aprendizaje, impactando, sobre todo, en el funcionamiento de las instituciones de educación formal (Vicenzi, 2020), ante el obligado cierre de miles de centros a lo largo y ancho del planeta.

Dada la situación anterior, las TIC se han posicionado como el medio que permite la continuidad de los procesos educativos formales en las universidades, tanto de manera autónoma como colectivamente (Sandoval, 2020), dejando entrever, a su vez, la importancia del aprendizaje informal. Al respecto, múltiples recursos permiten el acceso a gran cantidad de información, la creación de nuevo contenido, así como su rápida difusión y socialización entre los usuarios, procurando el respeto a las medidas sanitarias tomadas a nivel mundial que recomiendan el distanciamiento social. En este sentido tanto los procesos educativos formales y, especialmente los informales (Souto-Seijo, Estévez, Romero y González-Sanmamed, 2020), se ven altamente favorecidos gracias al uso de las TIC, sobre todo en situaciones de crisis como la vivida actualmente.

Con el impulso de la Sociedad del Conocimiento, se han generado cambios paradigmáticos en los procesos educativos. Las nuevas ecologías de aprendizaje aportan un sentido de mayor envergadura a estos procesos (Monsalve-Lorente y Aguasanta-Regalado, 2020), con elementos derivados desde sistemas complejos y dinámicos interrelacionados (González-Sanmamed, Sangrà, Souto-Seijo y Estévez, 2020; Souto-Seijo, 
Aprender conectados: un estudio sobre las redes personales de aprendizaje de estudiantes universitarios

Jose Antonio García-Martínez, Graciela Herrera-Villalobos y Manuel Arturo FallasVARGAS

Estévez, Iglesias y González-Sanmamed, 2020). Estos ecosistemas destacan, entre otros aspectos, por la promoción de las interacciones con diversas personas (González-Sanmamed, Estévez, Souto-Seijo \& MuñozCarril, 2020) así como el reconocimiento de otros tipos de aprendizaje complementarios a la educación formal. Aspectos que fomentan la creación de comunidades educativas, no solo con otros estudiantes, sino con docentes y profesionales en diferentes áreas que enriquecen y expanden los aprendizajes y las reflexiones.

Al respecto, los denominados entornos personales de aprendizaje (PLE) se asumen como parte de estos elementos facilitadores del aprendizaje. Y gracias al avance y uso generalizado de las tecnologías -especialmente de la web 2.0-, se han amplificado sustancialmente los PLE, promoviendo la autonomía e impulsando un rol activo en las construcciones de aprendizaje, tanto en los ámbitos formales como informales (Fernández, Sánchez y Heras, 2020). Aunque no existe un consenso por parte de la comunidad científica en torno al concepto de PLE (Ordaz y González-Martínez, 2020), se puede identificar una doble vertiente desde la que se alude a los elementos tecnológicos y pedagógicos. En este sentido, destaca la propuesta de Castañeda y Adell, (2013) que consideran los PLE como un entramado de herramientas, mecanismos, actividades y conexiones que cada persona utiliza para aprender. Igualmente, los PLE quedan caracterizados por la flexibilidad de incorporar aquellos recursos que más se adapten a las circunstancias de aprendizaje del usuario (Castañeda, Tur y Torres-Kompen, 2019). En este sentido, cabe destacar también que el análisis de los PLE se propone una estructura basada en tres componentes: buscar información, crear contenido y compartir información (Castañeda y Adell, 2013).

Estudios previos, indican que el estudiantado universitario utiliza en mayor medida las herramientas relacionadas con la búsqueda de información (García-Martínez y González-Sanmamed, 2017; Tirado y Roque, 2019) y, en menor medida, los recursos destinados a compartir información (García-Martínez y González-Sanmamed, 2020). Siendo el componente menos desarrollado el referido a la generación de contenido (García-Martínez y González-Sanmamed, 2019). A nivel general hay que señalar que en las investigaciones advierten de un uso poco efectivo de las herramientas tecnológicas por parte de la población estudiantil universitaria, lo que puede suponer una merma en el desarrollo de sus PLE (Yen, Tu, Sujo-Montes, Harati y Rodas, 2019). 
Dentro de este complejo ecosistema, cobra especial relevancia el componente relativo a compartir información, a partir del cual se torna importante la creación y desarrollo de redes personales de aprendizaje (PLN) como un entorno social para generar procesos de aprendizaje colaborativo y de autoaprendizaje, donde las diversas fuentes de información pueden nutrirse de manera recíproca a través de múltiples interacciones en línea. En este sentido, las PLN quedan caracterizadas por el rol activo que cada persona puede ejercer para el manejo de datos en continua expansión (Green, 2020) aprovechando la potencialidad de los diversos recursos disponibles y facilitando un aprendizaje autónomo y a lo largo de la vida (González-Sanmamed et al., 2020).

Aunque es notable la capacidad del ser humano para aprender en diferentes situaciones, es importante la ruptura de barreras espaciales y temporales para enriquecer la interacción social, dado que esta impacta profundamente en la construcción y resignificación de los aprendizajes (García-Martínez y González-Sanmamed, 2020). En este sentido, múltiples recursos permiten la participación en diálogos, foros, discusiones y congresos académicos digitales facilitando la interacción entre las diferentes perspectivas existentes y traspasando las fronteras socioculturales (Castañeda y Adell, 2013). En cuanto a las herramientas más usadas para la creación de PLN, destacan los blogs, gestores de correo electrónico, sitios web y grupos de noticias, a través de los cuales se puede generar información rápida y actualizada (Green, 2020). Sin embargo, para aprovechar una experiencia de mayor riqueza, múltiples recursos permiten romper el entorno de espacio y tiempo para interactuar con más personas y profesionales del área de interés, por ejemplo, a través de redes sociales como Twitter, Linked-in, entre otros (Green, 2020).

Desde hace unas décadas, la web 2.0 se torna como un nuevo paradigma que reconceptualiza la manera de utilizar internet (Gutiérrez-Porlán, Román-García y Sánchez-Vera, 2018), ya que ofrece innumerables recursos y aplicaciones que promueven la interrelación y participación de manera activa de las personas usuarias. Su uso deriva en una exposición constante a un sinnúmero de información que enriquece sustancialmente los aprendizajes, así como la creación y el aumento de enlaces y contactos importantes para continuar con la construcción y distribución de contenido (Palacio, 2020).

En la actualidad, los medios de comunicación digitales han aumentado la práctica de incluir en sus plataformas la participación impres- 
Aprender conectados: un estudio sobre las redes personales de aprendizaje de estudiantes universitarios

Jose Antonio García-Martínez, Graciela Herrera-Villalobos y Manuel Arturo FallasVARGAS

cindible de los usuarios para difundir información (Sixto-García, LópezGarcía y Toural-Bran, 2020). Aspecto que resalta la importancia de la cocreación como término que cada vez cobra mayor relevancia en los contextos actuales y en las interacciones facilitadoras de la comunicación. En este sentido, las PLN promueven esta creación conjunta de contenido y, especialmente, la rápida difusión a través de los diferentes nodos que las integran.

Resulta importante destacar que el conocimiento de las herramientas TIC no es suficiente para garantizar una adecuada interacción en las diferentes comunidades y espacios digitales, sino que se requiere del desarrollo de habilidades y actitudes específicas que promuevan la participación asertiva por parte de las personas usuarias (Gutiérrez- Porlán et al., 2018). También se recomienda propiciar el protagonismo de los estudiantes en la construcción de aprendizajes que involucren las interacciones y la información que aportan las diferentes comunidades educativas (Green, 2020).

EI PLE propicia que cada persona logre una autorregulación en el aprendizaje que implica el establecimiento de objetivos (Yen, et al., 2019), así como el desarrollo de procesos de autoevaluación (GutiérrezPorlán et al., 2018), siendo estas actitudes indispensables para potenciar un buen rendimiento académico. Al respecto, la construcción de PLN, y el uso de recursos de forma eficiente, permiten el desarrollo de mecanismos como dialogar, reflexionar, construir, deconstruir y reconstruir conocimiento, que contribuirán al enriquecimiento de los aprendizajes (Castañeda y Adell, 2013). Estas mejoras en el aprendizaje repercutirán en el rendimiento académico en la educación formal y en la adquisición de competencias indispensables para el mundo laboral desde otros ámbitos (Souto-Seijo et al., 2020). Estudios al respecto, señalan las interacciones sociales y familiares como un factor destacable, no solamente en el rendimiento académico, sino para la permanencia en la universidad (Velázquez y González, 2017).

Con la integración de plataformas digitales colectivas se propicia el crecimiento exponencial de las comunidades de aprendizaje, sobre todo en las personas universitarias, una de cuyas características será precisamente la participación proactiva. Este tipo de comunidades suponen mayor apoyo ante interrogantes que surgen en el proceso de construcción de los aprendizajes (Garay-Ruiz, Tejada y Romero-Andonegi, 2017). Es innegable que los procesos de enseñanza-aprendizaje comprometidos 
con el desarrollo de las PLN son más eficaces en el logro de propósitos valiosos como los relativos a la promoción de la autonomía, el desarrollo de un ejercicio activo de solución de conflictos, la construcción colectiva de aprendizajes, la generación de procesos reflexivos de autorreflexión, habilidades comunicativas asertivas, etc. (Castañeda y Adell, 2013). Igualmente, las PNL ayudarán a situar a los estudiantes como protagonistas del aprendizaje (García-Martínez y González-Sanmamed, 2017) y a incorporar las habilidades que surgen de ambientes informales (González-Sanmamed et al., 2019; Souto-Seijo et al., 2020).

\section{Marco empírico}

\section{Objetivos}

Teniendo en cuenta los aspectos comentados en los párrafos anteriores, el propósito general de este trabajo, que pertenece a un proyecto de mayor envergadura, es analizar las interacciones en las que participa el estudiantado de último año de carrera a través de herramientas tecnológicas durante el proceso de adquisición de aprendizajes. Específicamente se han propuestos los siguientes objetivos:

1. Identificar las fuentes y la frecuencia de interacción que utilizan los estudiantes.

2. Determinar la frecuencia de uso de las principales herramientas tecnológicas utilizadas para la interacción.

3. Identificar diferencias en las interacciones de acuerdo con la variable rendimiento académico.

\section{Metodología}

Teniendo en cuenta tanto supuestos teóricos como metodológicos de acercamiento al objeto de estudio, la presente investigación se realizó desde un enfoque cuantitativo. Concretamente, el estudio empírico se desarrolló a través de un diseño ex post facto. Además, atendiendo al momento de recogida de datos, el estudio responde a un diseño transaccional. En cuanto al alcance de la investigación, señalar que es de tipo descriptivo (Hernández, Fernández y Baptista, 2014). 
Aprender conectados: un estudio sobre las redes personales de aprendizaje de estudiantes universitarios

Jose Antonio García-Martínez, Graciela Herrera-Villalobos y Manuel Arturo FallasVARGAS

\section{Población y muestra}

La población ( $\mathrm{N}=3165)$ quedó compuesta por estudiantes universitarios, de último año de carrera, de la Universidad Nacional (UNA) de Costa Rica, tanto del Campus Omar Dengo como del Benjamín Núñez.

La muestra $(n=1187)$ se seleccionó de forma probabilística, respondiendo al tipo estratificado (Hernández et al., 2014). Se han tomado como referencia las siete áreas de estudio reconocidas en la UNA y distribuidas en las diferentes facultades. El estudio se llevó a cabo con un total de 51 carreras distintas. Para obtener el tamaño muestral, se utilizó la fórmula para poblaciones finitas propuesta por Arnal, del Rincón y Latorre (1992): error de muestreo 3\%, un nivel de confianza de 95\%, y la proporción esperada ( $p=5 \%)$.

En cuanto a la edad del grupo participante varía en un rango entre los 20 y 57 años $(M=24$; DS= 4.18). Del total de la muestra el $64.1 \%$ son mujeres y el restante $35.9 \%$ hombres. En la Figura 1 se observa la distribución porcentual por área de estudio.

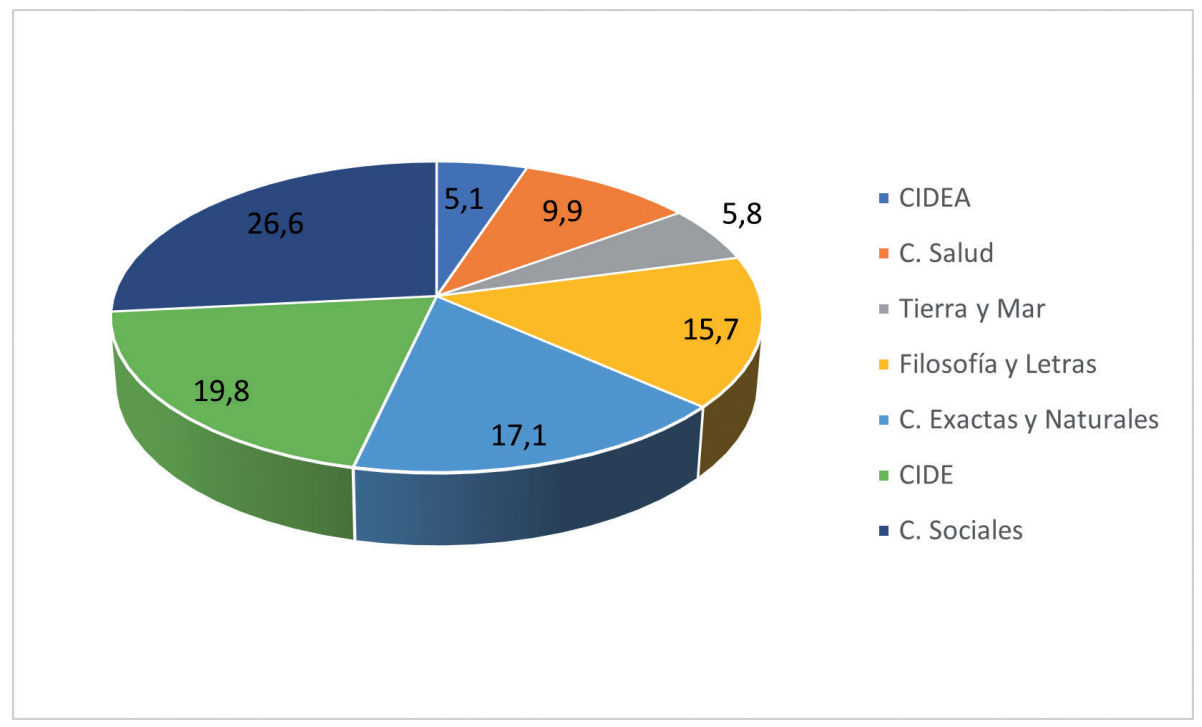

Figura 1. Distribución porcentual de la muestra por área de estudio Nota: CIDEA: Centro de Investigación, Docencia y Extensión Artística; CIDE: Centro de Investigación y Docencia en Educación. 


\section{Técnicas e instrumentos de recogida de datos}

Cabe destacar que este trabajo pertenece a una investigación mucho más amplia en la que se utilizó la técnica de encuesta para la recolección de datos. El instrumento aplicado fue elaborado ad hoc y quedó estructurado en 7 bloques. En este artículo se presentan los datos relacionados con el bloque de interacciones. La escala en cuestión responde al tipo Likert compuesta por 30 afirmaciones relacionadas con las conexiones que utilizan dentro de sus PLE (ej.: "profesores/as") así como la frecuencia de uso de las TIC que utilizan como medio de interacción (ej.: "redes sociales"). Las opciones de respuesta varían en un rango de 5 puntos (desde $1=$ nada, hasta $5=$ muchísimo).

Se realizó la prueba de consistencia interna para comprobar la fiabilidad de la escala, obteniendo coeficientes favorables de .918 en alfa de Cronbach y .922 en omega.

\section{Procedimiento y análisis}

En primer lugar, y posterior a una exhaustiva revisión de literatura, se elaboró un borrador de cuestionario y se sometió al filtro del grupo de investigación. Posteriormente, como método de validación de contenido fue revisado por expertos, tanto del área de Tecnología Educativa como de Metodología de Investigación, en todos los casos con grado de Doctor. El panel de expertos quedó compuesto por 20 profesionales con grado de Doctor de diferentes universidades costarricenses y españolas. Atendiendo a las diferentes observaciones realizadas por los revisores, se elaboró la primera versión de la escala. El instrumento se aplicó por medio de un pilotaje a 45 estudiantes de último año de carrera de diferentes áreas de estudio. Esta prueba permitió, por un lado, definir la matriz de datos elaborada en un paquete estadístico. Por otro lado, se Ilevaron a cabo diferentes análisis estadísticos para comprobar el comportamiento de la escala en general y de cada uno de los ítems en particular. Igualmente se obtuvieron los primeros datos sobre la fiabilidad a través del Alfa de Cronbach y el coeficiente Omega por tratarse de una escala ordinal.

El cuestionario definitivo fue aplicado en las aulas de clase de las diferentes facultades a lo largo de dos meses. Cabe destacar los aspectos éticos tenidos en cuenta a lo largo del proceso de investigación, en par- 
Aprender conectados: un estudio sobre las redes personales de aprendizaje de estudiantes universitarios

Jose Antonio García-Martínez, Graciela Herrera-Villalobos y Manuel Arturo FallasVARGAS

ticular tanto durante la recolección de datos como en su análisis. En el instrumento se anexó una página donde se explicaron los objetivos de investigación, el tiempo aproximado que requería la cumplimentación del cuestionario, el tipo de tratamiento de los datos que se realizaría, y el carácter voluntario de su participación, garantizando que se podrían retirar del estudio sin tener que dar explicaciones previas. Por características de la muestra en cuanto a que son mayores de edad, el consentimiento informado se realizó de forma oral y la entrega del cuestionario completo implicaba el consentimiento para el uso de los datos con fines de la investigación.

Los datos fueron introducidos en el paquete estadístico SPSS v.21. Las pruebas estadísticas realizadas inicialmente fueron de tipo descriptivo (medidas de tendencia central y variabilidad). Igualmente, se lleva a cabo la prueba de consistencia interna Alpha de Cronbach y Omega, este último por tratarse de una escala ordinal, y su cálculo se realizó utilizando el programa JASP. Posteriormente, se generan pruebas para la verificar las diferencias entre grupos, concretamente a través de t de Student, y comprobando previamente la normalidad (Kolmogorov-Smirnov para los diferentes grupos) y la homogeneidad de varianzas (prueba de Levene).

\section{Resultados}

\section{Frecuencia de interacciones y empleo de herramientas TIC}

A continuación, se muestran los datos porcentuales y descriptivos de las frecuencias de interacción que utiliza el estudiantado durante el proceso de aprendizaje. Como se puede observar (Tabla 1), la mayor interacción se lleva a cabo entre el grupo de compañeros ( $M=3.20$, $D S=.76)$; seguido del profesorado $(M=2.97, D S=.74)$. En menor medida con grupos de profesionales de distintas áreas $(M=2.45, D S=.81)$; con familiares y amistades $(M=2.24, D S=.96)$ y con profesionales del área de estudio $(M=2.24, D S=.97)$.

En cuanto a las herramientas que utilizan como medio de interacción, los datos de la Tabla 1 muestran como la comunicación móvil es la más utilizada $(M=3.27, D S=.87)$. En segundo lugar, usan el correo electrónico $(M=3.16, D S=.91)$, seguido de las redes sociales en gene- 
ral $(M=2.98, D S=.96)$. En menor medida utilizan blogs y páginas webs $(M=2.50, D S=1.0)$; foros $(M=2.20, D S=.92)$, y muy poco las redes profesionales $(M=1.76$; $D S=.94)$, donde el $52.2 \%$ indica no utilizarlas nada y el $25.3 \%$ poco.

Tabla 1

Porcentajes y descriptivos de la frecuencia de interacciones y uso de herramientas

\begin{tabular}{lcccccccc}
\hline \multicolumn{10}{c}{ Nada Poco Moderado Mucho Muchísimo Media DS } \\
\hline Interacciones & \multicolumn{1}{c}{ D } \\
\hline Profesorado & 1.7 & 21.1 & 49.7 & 24.9 & 2.6 & 2.97 & .74 \\
Compañeros/as & .9 & 13.3 & 46.5 & 34.2 & 5.1 & 3.20 & .76 \\
Profesionales del área & 22.1 & 40.4 & 24.2 & 11.1 & 2.2 & 2.24 & .97 \\
Otros profesionales & 22.1 & 39.4 & 25.2 & 11.1 & 2.2 & 2.45 & .81 \\
Familia y amistades & 11.3 & 39.9 & 38.4 & 8.7 & 1.7 & 2.24 & .96 \\
\hline Herramientas & & & & & & & \\
\hline Redes Sociales & 6.8 & 21.6 & 37.6 & 27.8 & 6.2 & 2.98 & .96 \\
Comunicación móvil & .8 & 15.8 & 42.6 & 29.9 & 10.9 & 3.27 & .87 \\
Redes profesionales & 52.2 & 25.3 & 14.8 & 6.4 & 1.3 & 1.76 & .94 \\
Foros & 26.0 & 36.0 & 26.6 & 10.5 & .9 & 2.20 & .92 \\
Blogs y páginas webs & 16.3 & 33.0 & 31.1 & 13.8 & 3.8 & 2.50 & 1.0 \\
Correo electrónico & 1.9 & 21.8 & 36.8 & 31.1 & 8.4 & 3.16 & .91 \\
\hline
\end{tabular}

Específicamente, el colectivo de estudiantes se relaciona con el profesorado a través de diferentes herramientas (Figura 2). La más utilizada es el correo electrónico, donde el $86.1 \%$ indica hacerlo mucho o muchísimo. Igualmente son muy utilizadas las comunicaciones móviles como WhatsApp (52.5\%). Otros recursos como los blogs y foros son de uso moderado. Nada o poco utilizadas $(74.5 \%)$ para comunicarse con el profesorado son las redes profesionales, donde solamente el $11.9 \%$ dice utilizarlas mucho o muchísimo. Por último, también las redes sociales en general son utilizadas poco o muy poco $(60.7 \%)$ y solamente el $18.7 \%$ lo hace con elevada frecuencia. 
Aprender conectados: un estudio sobre las redes personales de aprendizaje de estudiantes universitarios

Jose Antonio García-Martínez, Graciela Herrera-Villalobos y Manuel Arturo FallasVARGAS

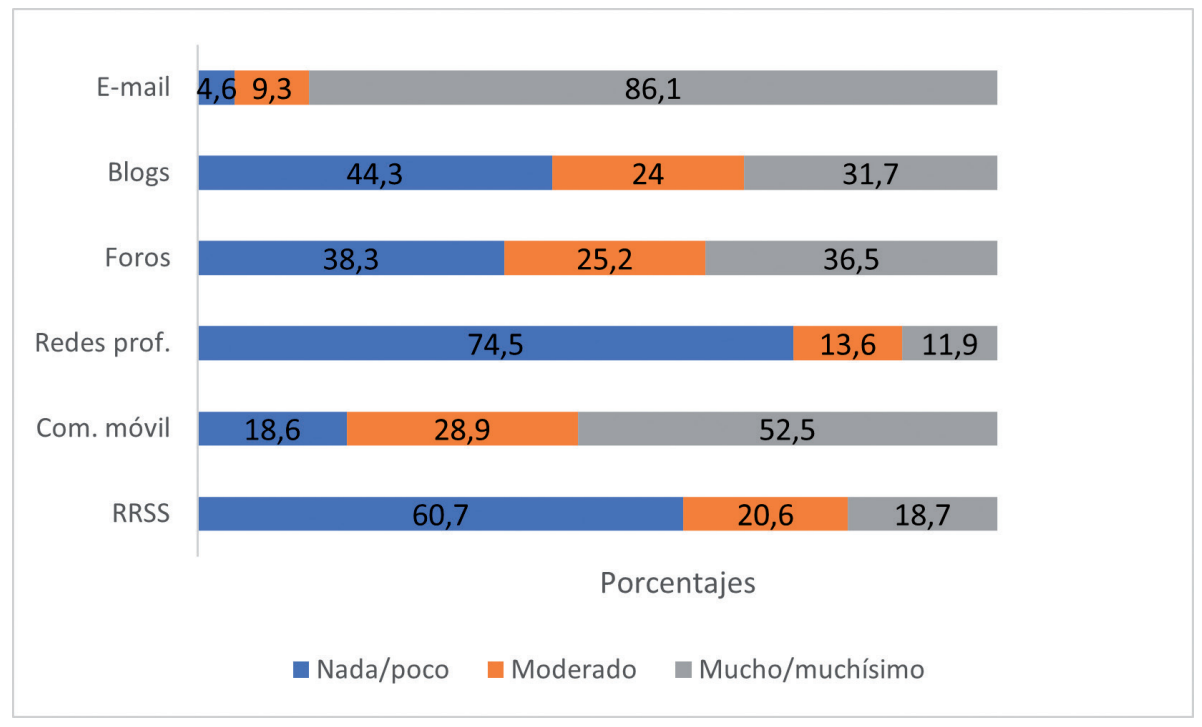

Figura 2. Herramientas de interacción con el profesorado

Entre el grupo de pares, como se observa en la Figura 3 el estudiantado utiliza en mayor medida la comunicación móvil, indicando utilizarla mucho o muchísimo el $88.1 \%$, y solamente el $3.7 \%$ dice no utilizarla. Otro recurso muy utilizado es el correo electrónico $(77.3 \%)$, seguido de las redes sociales, las cuales se emplean mucho o muchísimo por el $55.6 \%$, aunque cabe destacar un $23.8 \%$ que dice utilizarlas nada o poco. La herramienta de interacción menos utilizada son las redes profesionales, un $75.8 \%$ indica que las usa nada o poco, y solamente el $12.4 \%$ las utiliza con asiduamente. Al igual que con el profesorado, el empleo de blogs y foros está relegado a un uso moderado, donde la mitad aproximadamente indica que las usa nada o poco. 
Aprender conectados: un estudio sobre las redes personales de aprendizaje de estudiantes universitarios Jose Antonio García-Martínez, Graciela Herrera-Villalobos y Manuel Arturo Fallas-

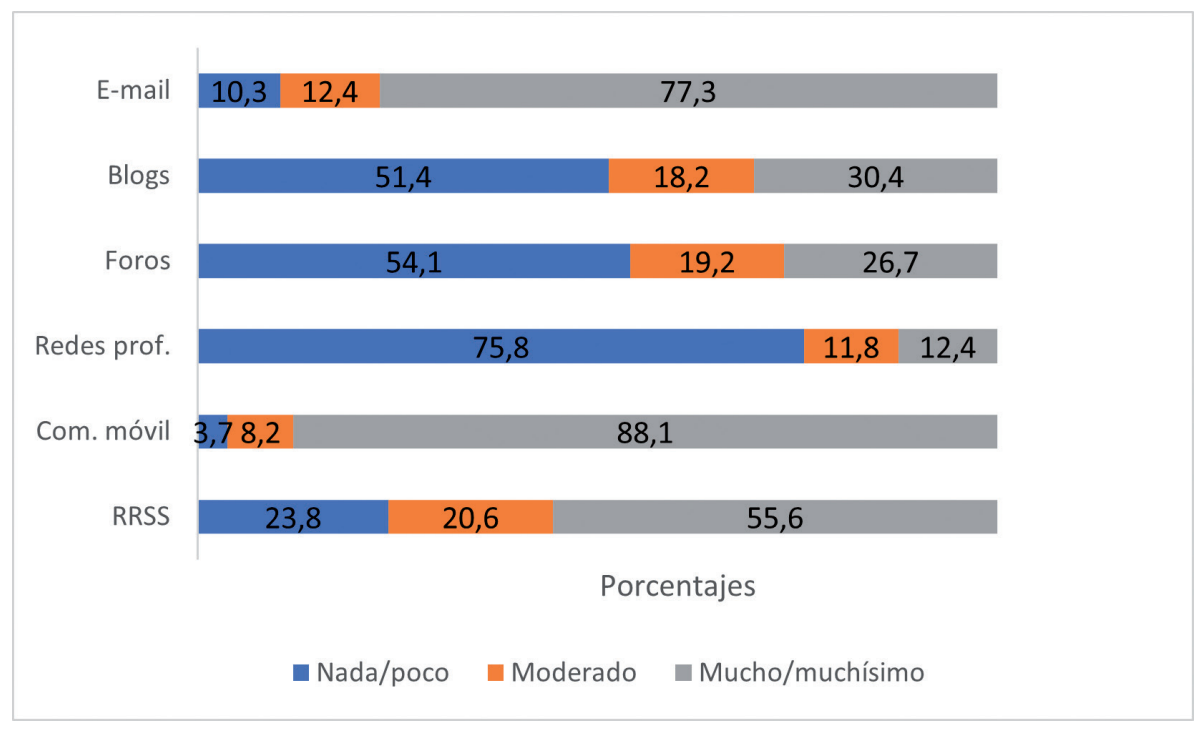

Figura 3. Herramientas de interacción con compañeros/as

En cuanto a las interacciones con profesionales del área de estudio, cabe destacar el escaso uso de recursos para tal fin. Como se observa en la Figura 4, sigue siendo el correo el medio más utilizado, donde un $43.9 \%$ lo hace con mucha frecuencia. Seguidamente, utilizan con mucha frecuencia, blogs (36.5\%), redes sociales (35.2\%) y comunicación móvil (31.6\%). Sin embargo, como ya se ha comentado, esta interacción se caracteriza por la baja frecuencia de uso. Al respecto indican utilizar nada o poco las redes profesionales (70.5\%); foros (54.1\%); comunicación móvil (469\%) y redes sociales (44.6\%). 
Aprender conectados: un estudio sobre las redes personales de aprendizaje de estudiantes universitarios

Jose Antonio García-Martínez, Graciela Herrera-Villalobos y Manuel Arturo FallasVARGAS

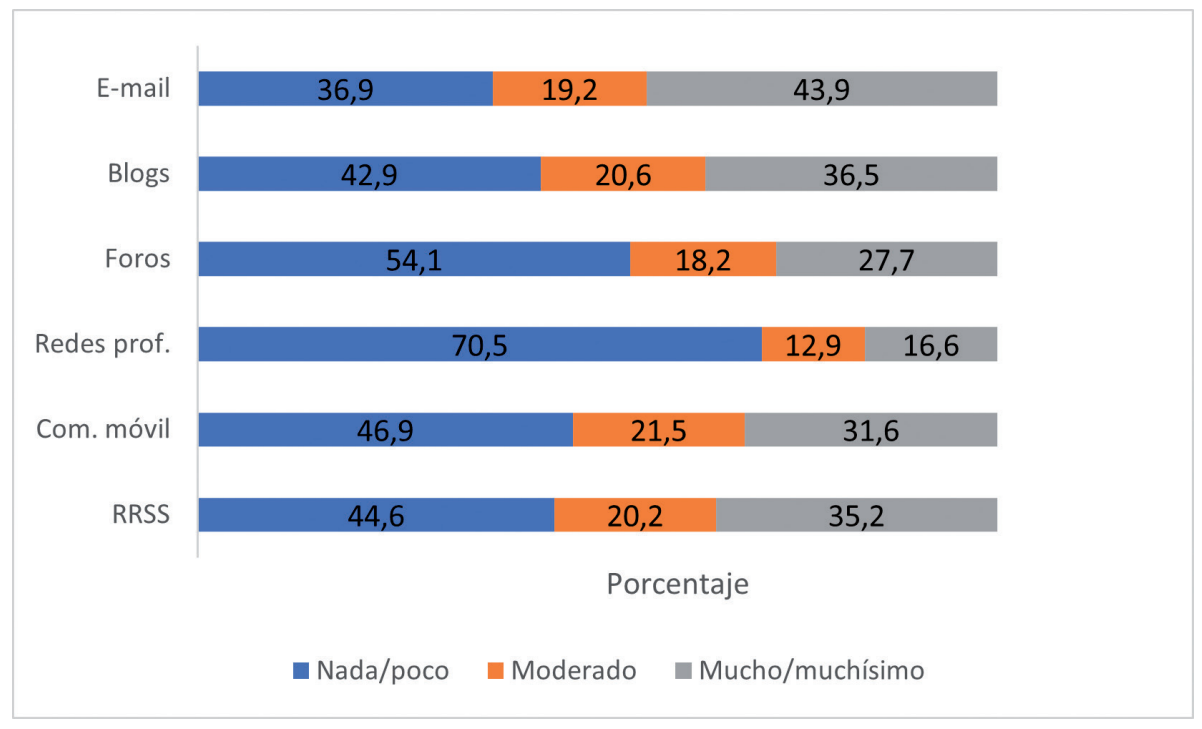

Figura 4. Herramientas de interacción con profesionales del área de estudio

\section{Diferencias en las interacciones de acuerdo con la variable rendimiento académico}

Se hicieron diferentes preguntas relacionadas con el rendimiento académico. Cabe destacar que la nota promedio de la muestra es de 8.3 (DS=.71). De estos, el 56.5\% indica que nunca ha suspendido asignaturas, mientras el restante $43.5 \%$ sí ha suspendido alguna vez.

Para identificar las diferencias en las interacciones a través de las herramientas TIC según la variable rendimiento académico, se generaron dos grupos, aquellos que nunca han suspendido materias y los que sí lo han hecho. Antes de comparar las medias de ambos grupos, se llevó a cabo la prueba de contraste Kolmogorov-Smirnov para evaluar la normalidad, obteniendo un nivel " $p$ " no significativo ( $p>.05$ ) para los grupos descritos. Igualmente, se realizó la prueba de Levene (p-valor>.05) para comprobar el supuesto de homogeneidad de varianzas. Los datos de la prueba t de Student (tabla 2), muestran que los estudiantes que nunca han suspendido materias obtienen puntuaciones medias más altas en las interacciones, siendo significativas en el caso del profesorado, el grupo de compañeros y profesionales del área de estudio. No se han encontrado diferencias en las interacciones con otros profesionales ni en las desarrolladas con la familia y amigos. 
Aprender conectados: un estudio sobre las redes personales de aprendizaje de estudiantes universitarios

Jose Antonio García-Martínez, Graciela Herrera-Villalobos y Manuel Arturo Fallas-

Tabla 2

Estadísticos de grupo y resultados de las pruebas t de Student con relación al rendimiento académico

\begin{tabular}{|c|c|c|c|c|c|c|}
\hline \multirow{2}{*}{ Interacciones } & \multicolumn{2}{|c|}{ Estadísticos de grupo } & \multicolumn{4}{|c|}{ Prueba T para la igualdad de medias } \\
\hline & Edad & Media & DS & $t$ & gl & Sig. (bilateral) \\
\hline \multirow{2}{*}{ Profesorado } & NR & 3.02 & .74 & \multirow{2}{*}{-2.265} & \multirow{2}{*}{1171} & \multirow{2}{*}{.024} \\
\hline & $\mathrm{R}$ & 2.86 & .72 & & & \\
\hline \multirow{2}{*}{ Compañeros/as } & NR & 3.22 & .76 & \multirow{2}{*}{-2.005} & \multirow{2}{*}{1162} & \multirow{2}{*}{.037} \\
\hline & $\mathrm{R}$ & 3.10 & .76 & & & \\
\hline \multirow{2}{*}{$\begin{array}{l}\text { Profesionales área } \\
\text { de estudio }\end{array}$} & NR & 2.26 & .98 & \multirow{2}{*}{-1.876} & \multirow{2}{*}{1170} & \multirow{2}{*}{.043} \\
\hline & $\mathrm{R}$ & 2.16 & .94 & & & \\
\hline \multirow{2}{*}{$\begin{array}{l}\text { Otros } \\
\text { profesionales }\end{array}$} & NR & 2.26 & .98 & \multirow{2}{*}{.898} & \multirow{2}{*}{1172} & \multirow{2}{*}{.051} \\
\hline & $\mathrm{R}$ & 2.21 & .82 & & & \\
\hline \multirow{2}{*}{ Familia y amigos } & NR & 2.48 & .80 & \multirow{2}{*}{-1.332} & \multirow{2}{*}{1173} & \multirow{2}{*}{.064} \\
\hline & $\mathrm{R}$ & 2.42 & .82 & & & \\
\hline
\end{tabular}

Nota: $N R=$ nunca ha reprobado materias; $R=$ ha reprobado materias.

\section{Discusión y Conclusiones}

Estudiar las interacciones desarrolladas a través de los recursos tecnológicos por parte del alumnado universitario de último año de carrera, aporta luces al engranaje complejo que supone el aprendizaje, especialmente ante los cambios que generan las TIC (Ordoñez et al., 2021), tanto en procesos educativos como en lo que respecta a sus implicaciones culturales y sociales. Por otro lado, los hallazgos cobran relevancia al tratarse de una población que de forma inminente se incorporará al mundo laboral y, particularmente, por su repercusión en la adquisición de habilidades y competencias necesarios para el desempeño de los diferentes roles profesionales (Souto-Seijo et al., 2020). Al respecto, la construcción consciente de los PLE, que implica conocer cómo y con quién aprendemos a través de los múltiples recursos disponibles (Castañeda y Adell, 2013) y, en especial, el desarrollo de PLN, supone un paso importante para alcanzar este objetivo.

Los datos muestran, de forma general, frecuencias moderadas de interacción con los recursos tecnológicos (Tabla 1). Destaca también la intensidad de las interacciones entre compañeros, seguida en porcentaje de importancia por la comunicación con el profesorado. Sin embargo, se obtienen bajas puntuaciones en las interacciones con profesionales 
Aprender conectados: un estudio sobre las redes personales de aprendizaje de estudiantes universitarios

Jose Antonio García-Martínez, Graciela Herrera-Villalobos y Manuel Arturo FallasVARGAS

de otras áreas, con la familia y amistades. Y sorprende que sean especialmente limitadas las relaciones con profesionales del área de estudio. Estos hallazgos, reflejan, pon un lado, el nexo generado entre la educación formal y los procesos de aprendizaje gracias a las TIC (Sandoval, 2020). Sin embargo, estas moderadas puntuaciones pueden suponer un detrimento de las ecologías de aprendizaje caracterizadas por la interrelación con diversas y amplias fuentes de información (González-Sanmamed et al., 2020). Igualmente, resulta preocupante que más allá de la educación formal, las PLN del estudiantado no aprovechen las múltiples posibilidades que aportaría la incorporación, en mayor medida, de profesionales del área de estudio que repercutan en la adquisición de aprendizajes informales (Fernández et al., 2020), así como de las habilidades y competencias que se derivan de estos.

Aunque se parte del carácter flexible y personal de los PLE, donde cada estudiante puede hacer uso de los recursos de acuerdo con su estilo de aprendizaje (Castañeda et al., 2019), los datos dejan entrever (Tabla 1) que las herramientas más utilizadas para la interacción son la comunicación móvil, seguida del correo electrónico y, en menor medida y con puntuaciones moderadas las redes sociales, coincidiendo parcialmente con otras investigaciones (Green, 2020), en las que se hace énfasis en los blogs y otros sitios web como recursos potenciales de las PLN. Precisamente, el estudiantado utiliza con frecuencia moderada los blogs y páginas webs, y con bajas frecuencias foros y especialmente redes profesionales, estas últimas nada utilizadas por más de la mitad de la muestra. Al respecto, aunque estudios previos (García-Martínez y González-Sanmamed, 2020) indican que el componente de compartir información y comunicación es uno de los más desarrollados dentro de los PLE del alumnado universitario, queda en evidencia un uso moderado de recursos. Cabe indicar que estas limitaciones pueden deberse a la poca efectividad a la hora de utilizar las TIC para la adquisición de aprendizajes (Yen et al., 2019) y al predominio de interacciones tradicionales que no implican el uso de recursos de la web 2.0 (Gutiérrez-Porlán et al, 2018), lo que puede generar una merma en las PLN.

De acuerdo con los resultados obtenidos de las pruebas de comparación entre grupos (Tabla 2), se han encontrado diferencias significativas en las fuentes de interacción de acuerdo con la variable rendimiento académico. Los datos muestran que aquellos estudiantes que nunca han suspendido materias son los que interactúan en mayor medida a través 
del uso de las TIC y, concretamente, desarrollan con más intensidad la comunicación con los compañeros, docentes y profesionales en el área de estudio. Este hallazgo pone de relieve el carácter social del aprendizaje y la importancia de las interacciones como impulsoras a favor del rendimiento académico y la permanencia en la universidad (Velázquez y González, 2017). Al respecto, el PLE y particularmente las PLN son aliadas en procesos para el establecimiento de metas, la autorregulación del aprendizaje (Yen, et al., 2019), así como en el desarrollo de procesos de autoevaluación (Gutiérrez-Porlán et al., 2018), todos ellos aspectos claves en el rendimiento académico.

En la misma línea, la facilidad de acceso a múltiples interacciones desde cualquier dispositivo que cuente con conexión a internet no se limita ni a un horario académico concreto (García-Martín y Cantón-Mayo, 2019) ni se constriñe a las conexiones enmarcadas en los límites tradicionales de la educación formal. La construcción consciente de PLN, permite una rápida retroalimentación (Garay et al., 2017), lo que contribuye a un avance más fluido en los procesos de aprendizaje (Gutiérrez-Porlán et al., 2018), así como en el intercambio de opiniones y reflexiones, lo cual genera un enriquecimiento a un nivel más complejo y significativo del aprendizaje, impactando positivamente en el rendimiento académico (García-Martín y Cantón-Mayo, 2019).

Las nuevas demandas que surgen en torno a la sociedad del conocimiento requieren del uso de las TIC para favorecer, cualitativa y cuantitativamente, las interacciones. La creación de PLN supone una opción complementaria para el estudiantado universitario de cara a nutrir las conexiones con compañeros, el profesorado, y los profesionales, tanto del campo de estudio como de otros contextos, derribando las fronteras espaciales y temporales. Igualmente, es importante tanto el reconocimiento de los aprendizajes informales, como la necesidad de generar conciencia en torno a las habilidades y competencias derivadas de estos. Estos pasos son vitales para entender los transcendentales cambios y las posibilidades que actualmente posibilitan el aprendizaje autodirigido y a lo largo de la vida.

En cuanto a limitaciones, cabe destacar que el estudio se lleva a cabo con estudiantes universitarios de la Universidad Nacional de Costa Rica, por lo que sería interesante ampliar el estudio a otros contextos analizar y comparar los resultados. Igualmente, este trabajo responde a un estudio transaccional con estudiantes de último año de carrera, por lo 
Aprender conectados: un estudio sobre las redes personales de aprendizaje de estudiantes universitarios

Jose Antonio García-Martínez, Graciela Herrera-Villalobos y Manuel Arturo FallasVARGAS

que se sugiere promover investigaciones longitudinales con otros cursos para comprobar la incorporación de recursos tecnológicos a lo largo de los años de carrera. Por último, a la luz de los resultados obtenidos se propone un estudio con un diseño cualitativo que permita profundizar en las interacciones.

\section{Referencias}

Arnal, J., Del Rincón, D., y Latorre, A. (1992). Investigación educativa: fundamentos y metodología. Barcelona: Labor.

Castañeda, L., Tur, G., y Torres-Kompen, R. (2019). Impacto del concepto PLE en la literatura sobre educación: la última década. RIED. Revista Iberoamericana de Educación a Distancia, 22(1), 221-241.doi: http://dx.doi.org/10.5944/ried.22.1.22079

Castañeda, L., y Adell, J. (2013.) Entornos Personales de Aprendizaje: claves para el ecosistema educativo en red. Alcoy: Marfil.

Fernández, M., Sánchez, A., y Heras, D. (2020) Las actividades de enseñanza-aprendizaje en el Espacio Europeo de Educación Superior las actividades prácticas con herramientas web 2.0. Revista Académia y Virtualida,. 13(1), 61-79. doi: https://doi. org/10.18359/ravi.4260

Garay-Ruiz, U., Tejada, E., y Romero-Andonegi, A. (2017). Rendimiento y satisfacción de estudiantes universitarios en una comunidad en línea de prácticas. Revista mexicana de investigación educativa., 22(75), 1239-1256. Recuperado de https:// bit.ly/35KtlHu

García-Martín, S. y Cantón-Mayo, I. (2019). Uso de tecnología y rendimiento académico en estudiantes adolescentes. Revista Científica de Educomunicación, 59(28), 73-81. doi: https://doi.org/10.3916/C59-2019-07

García-Martínez, J.A., y González-Sanmamed, M. (2017). Entornos personales de aprendizaje de estudiantes universitarios costarricenses de educación: análisis de las herramientas de búsqueda de información. Revista de Investigación Educativa, 35(2), 389-407. doi: https://doi.org/10.6018/rie.35.2.253101

García-Martínez, J.A., y González-Sanmamed, M. (2019). Cómo generan y gestionan contenidos los estudiantes de educación de Costa Rica: una contribución al estudio de su entorno personal de aprendizaje. Digital Education Review, 36, 15-35. Recuperado de https://revistes.ub.edu/index.php/der/article/view/22274

García-Martínez, J. A., y González-Sanmamed, M. (2020). La comunicación y la interacción como aspectos clave de los entornos personales de aprendizaje: Perspectiva de estudiantes costarricenses de educación. Revista Electrónica Educare, 24(3), 1-20. doi: http://doi.org/10.15359/ree.24-3.5

González-Sanmamed, M., Muñoz-Carril, P. C., y Santos-Caamaño, F. J. (2019). Key components of learning ecologies: A Delphi assessment. British Journal of Educational Technology, 5(4), 1639-1655. doi: https://doi.org/10.1111/bjet.12805

González-Sanmamed, M., Estévez, I., Souto-Seijo, A., y Muñoz-Carril, P.C. (2020) Digital 
Aprender conectados: un estudio sobre las redes personales de aprendizaje de estudiantes universitarios

Jose Antonio García-Martínez, Graciela Herrera-Villalobos y Manuel Arturo Fallas-

VARGAS

learning ecologies and professional development of university professors. Comunicar, 28(62), 9-18. doi: https://doi.org/10.3916/C62-2020-01

González-Sanmamed, M., Sangrà, A., Souto-Seijo, A., y Estévez, I. (2020). Learning ecologies in the digital era: challenges for higher education. Publicaciones, 50(1), 83-102. doi: 10.30827/publicaciones.v50i1.15671

Green, C. L. (2020, 21 de diciembre). Personal Learning Networks: Defining and Building a PLN. Learning in the Digital Age [Página web]. Recuperado de https://open. library.okstate.edu/learninginthedigitalage/chapter/personal-learning-networks_defining-and-building-a-pln/

Gutiérrez-Porlan, I., Román- García., M., y Sánchez-Vera., M. (2018) Estrategias para la comunicación y el trabajo colaborativo en red de los estudiantes universitarios. Revista Científica de Educomunicación, 54(15),91-100. doi: https://doi.org/10.3916/ C54-2018-09

Hernández, R., Fernández, C., y Baptista, P. (2014). Metodología de la investigación. México: Editorial Mc Graw Hill.

Monsalve- Lorente, L., y Aguasanta-Regalado, E. (2020). Nuevas Ecologías del aprendizaje en el currículo: La era digital en la escuela. Revista Latinoamericana de Tecnología Educativa, 19(1). 139-154. doi: https://doi.org/10.17398/1695-288X.19.1.139

Ordaz, T., y González-Martínez, J. (2020). Hacia una visión aglutinadora del concepto de PLE. Universitas Tarraconensis. Revista de Ciències de l'Educació, 2, 21-37. doi: https://doi.org/10.17345/ute.2020.2

Ordóñez, E., Vázquez-Cano, E., Arias-Sánchez, S. \& López-Meneses, E. (2021). Las Competencias en el uso de las Tecnologías de la Información y la Comunicación en el alumnado universitario. Píxel-Bit. Revista de Medios y Educación, 60, 153-167. doi: https://doi.org/10.12795/pixelbit.74860

Palacio, M. (2020). Aprendizaje colaborativo con tic y las excepciones y limitaciones al derecho de autor: Colombia. Revista Propiedad Intelectual, 29, 117-136. doi: https:// doi.org/10.18601/16571959.n29.05

Sandoval, C. (2020). La Educación en Tiempo del Covid-19 Herramientas TIC: El Nuevo Rol Docente en el Fortalecimiento del Proceso Enseñanza Aprendizaje de las Prácticas Educativa Innovadoras. Revista Tecnológica-Educativa Docentes 2.0, 9(2), 24-31. doi: https://doi.org/10.37843/rted.v9i2.138

Sixto-García, J., López-García, X., y Toural-Bran, C. (2020). Oportunidades para la cocreación de contenidos en los diarios nativos digitales. Profesional de la información, 29 (4), 1-19. doi: https://doi.org/10.3145/epi.2020.jul.26

Souto-Seijo, A., Estévez, I., Iglesias, V., y González-Sanmamed, M. (2020). Entre lo formal y lo no formal: un análisis desde la formación permanente del profesorado. Educar, 56(1), 97-107. doi: DOI: https://doi.org/10.5565/rev/educar.1095

Souto-Seijo, A., Estévez, I., Romero, P., y González-Sanmamed, M. (2020). Aprendizajes Formales, No Formales e Informales en la Era Digital: Contribuciones al Desarrollo Profesional Docente. En S. Oliveira e Sá, F. Freitas, P. Castro, M. González-Sanmamed, y A. P. Costa (Eds.), Investigación Cualitativa en Educación: avances y desafíos (Vol. 2, pp. 428-436). En https://doi.org/10.36367/ntqr.2.2020.428-436

Tirado, P., y Roque, M. del P. (2019). TIC y contextos educativos: frecuencia de uso y 
Aprender conectados: un estudio sobre las redes personales de aprendizaje de estudiantes universitarios

Jose Antonio García-Martínez, Graciela Herrera-Villalobos y Manuel Arturo FallasVARGAS

función por universitarios. Edutec. Revista Electrónica De Tecnología Educativa, 67, 31-47. doi: https://doi.org/10.21556/edutec.2019.67.1135

Uriguen-Aguirre, P., Vega- Jaramillo, F., Luna-Romero, Á. (2020). El uso de las TIC en el aprendizaje en la Universidad caso UTMACH. INNOVA Research Journal, 5(1), 3146. doi: https://doi.org/10.33890/innova.v5.n1.2020.1120

Velázquez, Y., y González, M. A. (2017). Factores asociados a la permanencia de estudiantes universitarios: caso UAMM-UAT. Revista de la educación superior. 46(184), 117-138. doi: https://doi.org/10.1016/j.resu.2017.11.003

Vicenzi, A. (2020) Del aula presencial al aula virtual universitaria en contexto de pandemia de COVID-19. Avances de una experiencia universitaria en carreras presenciales adaptadas a la modalidad virtual. Debate Universitario, 8(16), 67-71. Recuperado de $<$ http://200.32.31.164:9999/ojs/index.php/debate-universitario/article/view/238/242

Yen, C. J., Tu, C. H., Sujo-Montes, L. E., Harati, H., y Rodas, C. R. (2019). Using personal learning environment (PLE) management to support digital lifelong learning. International Journal of Online Pedagogy and Course Design (IJOPCD), 9(3), 13-31. doi: https://doi.org/10.4018/IJOPCD.201907010215

\section{Agradecimiento}

Este artículo se ha elaborado en el marco del proyecto de investigación titulado: "Ecologías de aprendizaje en la era digital: nuevas oportunidades para la formación del profesorado de educación secundaria" (ECO4LEARN-SE), parcialmente financiado por el Ministerio de Ciencia, Innovación y Universidades (Referencia RTI2018-095690-B-I00). 